\title{
INEQUALITIES OF DUNKL-WILLIAMS AND MERCER IN QUASI 2-NORMED SPACE
}

\author{
Katerina Anevska ${ }^{1}$ and Samoil Malčeski ${ }^{2}$
}

\begin{abstract}
C. Park [3] introduced the term of quasi 2-normed space, and further he has also proved few properties of quasi 2-norm. M. Kir and M. Acikgoz [4] gave the procedure for completing the quasi 2-normed space. Families of quasi-norms generated by quasi 2-norm are considered in [2] and are also proven few statements according to that ones. The inequalities of Dunkl-Williams, Mercer, Pečarić-Rajić and the sharp parallelepiped inequalities are fundamental in the theory of a 2-normed spaces. In quasi 2-normed spaces are proven, [1] and[2], the analogous inequalities of sharp inequalities and inequalities of Pečarić-Rajić type. In this paper will be considered inequalities, which are analogies to Dunkl-Williams and Mercer inequalities in quasi 2-normed spaces.
\end{abstract}

\section{INTRODUCTION}

S. Gähler (1965) gave the term of 2-norm ([11]). One of the axioms of 2-norm is the parallelepiped inequality, which is basic one in the theory of 2-normed spaces. Precisously this inequality, analogous as in normed spaces, C. Park has replaced by a new condition, and thus he actually obtained the following definition of quasi 2-normed space:

Definition 1 ([3]). Let $L$ be a real vector space and $\operatorname{dim} L \geq 2$. Quasi 2-norm is real function $\|\cdot, \cdot\|: L \times L \rightarrow[0, \infty)$ such that:

a) $\|x, y\| \geq 0$, for all $x, y \in L$ and $\|x, y\|=0$ iff the set $\{x, y\}$ is linearly dependent;

b) $\|x, y\|=\|y, x\|$, for all $x, y \in L$;

c) $\|\alpha x, y\|=|\alpha| \cdot\|x, y\|$, for all $x, y \in L$ and for each $\alpha \in \mathbf{R}$, and

d) it exists a constant $C \geq 1$ so that $\|x+y, z\| \leq C(\|x, z\|+\|y, z\|)$, holds for all $x, y, z \in L$.

An ordered pair $(L,\|\cdot, \cdot\|)$ is called as quasi 2-normed space. The smallest possible $C$ such that it satisfies the condition $d$ ) is called as modulus of concavity of quasi 2-norm $\|\cdot, \cdot\|$.

2010 Mathematics Subject Classification. Primary: 46B20 Secondary: 26D15

Key words and phrases. quasi 2-normed spaces, $(2, p)$-norm, inequality of Dunkl-Williams, 
Further, M. Kir and M. Acikgoz [4] have given few examples of trivial quasi 2normed spaces and have also considered the question about completing a quasi 2-normed space. In [2] is proven the following Lemma which is one of the basic while proving important inequalities in quasi 2-normed spaces.

Lemma 1. If $L$ is a quasi 2-normed space with modulus of concavity $C \geq 1$, then

$$
\left\|\sum_{i=1}^{n} x_{i}, z\right\| \leq C^{1+\left[\log _{2}(n-1)\right]} \sum_{i=1}^{n}\left\|x_{i}, z\right\|
$$

holds for each $n>1$ and for all $z, x_{1}, x_{2}, \ldots, x_{n} \in L$.

Further, C. Park gave a characterization of quasi 2-normed space, i.e. proved the following theorem.

Theorem 1 ([3]). Let $(L,\|\cdot, \cdot\|)$ be a quasi 2-normed space. It exists $p, 0<p \leq 1$ and an equivalent quasi 2-norm $\||\cdot \cdot|\|$ over $L$ so that

$$
\|x+y, z\|^{p} \leq\|x, z\|\left\|^{p}+\right\| y, z \|^{p},
$$

holds for all $x, y, z \in L$.

Definition 2 ([3]). The quasi 2-norm defined in Theorem 1 is called a $(2, p)-n o r m$, and the quasi 2-normed space $L$ is called a $(2, p)$-normed space.

\section{OUR RESULTS}

Theorem 2. Let $L$ be a quasi 2-normed space with modulus of concavity $C \geq 1$, and $V(z)$ be the subspace generated by vector $z$. The following inequality

$$
\left\|\frac{x}{\|x, z\|}-\frac{y}{\|y, z\|}, z\right\| \leq 4 C \frac{\|x-y, z\|}{\|x, z\|+\|y, z\|}+2(C-1) \frac{\max \{\|x, z\|,\|y, z\|\}}{\|x, z\|+\|y, z\|},
$$

holds true for each $z \in L \backslash\{0\}$ and for all $x, y \in L \backslash V(z)$.

Proof. Let $z \in L \backslash\{0\}$ and $x, y \in L \backslash V(z)$. Since definition 1 we get that

$$
\begin{aligned}
\|x, z\| \cdot \| \frac{x}{\|x, z\|}-\frac{y}{\|y, z\|}, z & \|\| x, z\|\cdot\| \frac{x}{\|x, z\|}-\frac{y}{\|x, z\|}+\frac{y}{\|x, z\|}-\frac{y}{\|y, z\|}, z \| \\
& \leq C\|x, z\| \cdot\left\|\frac{x}{\|x, z\|}-\frac{y}{\|x, z\|}, z\right\|+C\|x, z\| \cdot\left\|\frac{y}{\|x, z\|}-\frac{y}{\|y, z\|}, z\right\| \\
& \leq C\|x-y, z\|+C \mid\|y, z\|-\|x, z\| \| .
\end{aligned}
$$

Further, once again definition limplies that

$$
\|y, z\| \leq C\|y-x, z\|+C\|x, z\| \text { and }\|x, z\| \leq C\|x-y, z\|+C\|y, z\| .
$$

Therefore,

$$
\begin{aligned}
\|y, z\|-\|x, z\| & \leq C\|y-x, z\|+(C-1)\|x, z\| \\
& \leq C\|x-y, z\|+(C-1) \max \{\|x, z\|,\|y, z\|\}
\end{aligned}
$$


and

$$
\begin{aligned}
\|x, z\|-\|y, z\| & \leq C\|x-y, z\|+(C-1)\|y, z\| \\
& \leq C\|x-y, z\|+(C-1) \max \{\|x, z\|,\|y, z\|\},
\end{aligned}
$$

i.e. the inequality

$$
|\|y, z\|-\|x, z\|| \leq C\|x-y, z\|+(C-1) \max \{\|x, z\|,\|y, z\|\} .
$$

holds true. The inequalities (4) and (5) imply the inequality

$$
\|x, z\| \cdot\left\|\frac{x}{\|x, z\|}-\frac{y}{\|y, z\|}, z\right\| \leq 2 C\|x-y, z\|+(C-1) \max \{\|x, z\|,\|y, z\|\}
$$

The following inequality can be proven analogously

$$
\|y, z\| \cdot\left\|\frac{x}{\|x, z\|}-\frac{y}{\|y, z\|}, z\right\| \leq 2 C\|x-y, z\|+(C-1) \max \{\|x, z\|,\|y, z\|\} .
$$

Finally, if we add the inequalities (6) and (7) and so obtained inequality is divided by $\|x\|+\|y\|>0$ we get the inequality (3).

Theorem 3. Let $L$ be a $(2, p)$-normed space, $0<p \leq 1$, and $V(z)$ be a subspace generated by vector $z$. Then

$$
\left\|\frac{x}{\|x, z\|}-\frac{y}{\|y, z\|}, z\right\|^{p} \leq 2 \frac{\|x-y, z\|^{p}+\mid\|y, z\|-\|x, z\|^{p}}{\|x, z\|^{p}+\|y, z\|^{p}},
$$

for each $z \in L \backslash\{0\}$ and for all $x, y \in L \backslash V(z)$.

Proof. Definition 2, i.e. the properties of $(2, p)$-norm imply that each $z \in L \backslash\{0\}$ and all $x, y \in L \backslash V(z)$ satisfy the following

$$
\begin{gathered}
\|x, z\|^{p} \cdot\left\|\frac{x}{\|x, z\|}-\frac{y}{\|y, z\|}, z\right\|^{p}=\|x, z\|^{p}\left\|\frac{x}{\|x, z\|}-\frac{y}{\|x, z\|}+\frac{y}{\|x, z\|}-\frac{y}{\|y, z\|}, z\right\|^{p} \\
\leq\|x, z\|^{p}\left\|\frac{x}{\|x, z\|}-\frac{y}{\|x, z\|}, z\right\|^{p}+\|x, z\|^{p}\left\|\frac{y}{\|x, z\|}-\frac{y}{\|y, z\|}, z\right\|^{p} \\
\leq\|x-y, z\|^{p}+\|\| y, z\|-\| x, z \|\left.\right|^{p}
\end{gathered}
$$

and

$$
\begin{gathered}
\|y, z\|^{p} \cdot\left\|\frac{x}{\|x, z\|}-\frac{y}{\|y, z\|}, z\right\|^{p}=\|y, z\|^{p}\left\|\frac{x}{\|x, z\|}-\frac{x}{\|y, z\|}+\frac{x}{\|y, z\|}-\frac{y}{\|y, z\|}, z\right\|^{p} \\
\leq\|y, z\|^{p}\left\|\frac{x}{\|x, z\|}-\frac{x}{\|y, z\|}, z\right\|^{p}+\|y, z\|^{p}\left\|\frac{x}{\|y, z\|}-\frac{y}{\|y, z\|}, z\right\|^{p} \\
\leq\|x-y, z\|^{p}+\|\| y, z\|-\| x, z \|\left.\right|^{p} .
\end{gathered}
$$

Finally, if we add the inequalities (9) and (10) and the so obtained inequality we divide by $\|x\|^{p}+\|y\|^{p}>0$, we get the inequality (8).

Remark 1. The inequalities (3) and (8) are actually inequalities of Dunkl- Williams type in quasi-normed and $p$-normed space, $0<p \leq 1$, respectively.

Theorem 4. Let $L$ be a quasi 2 -normed space with modulus of concavity $C \geq 1$. The following statements are equivalent: 
1) For each $z \in L \backslash\{0\}$ and for all $x, y \in L \backslash V(z)$

$$
\left\|\frac{x}{\|x, z\|}-\frac{y}{\|y, z\|}, z\right\| \leq 2 C \frac{\|x-y, z\|}{\|x, z\|+\|y, z\|}+(C-1) \frac{\max \{\|x, z\|,\|y, z\|\}}{\|x, z\|+\|y, z\|} .
$$

2) If $x, y, z \in L$ are such that $\|x, z\|=\|y, z\|=1$, holds then

$$
\left\|\frac{x+y}{2}, z\right\| \leq C\|(1-t) x+t y, z\|+\frac{C-1}{2} \max \{1-t, t\},
$$

for each $t \in[0,1]$.

Proof. 1) $\Rightarrow 2$ ). Let assume that 1) is satisfied. Let $x, y, z \in L$ be such that

$$
\|x, z\|=\|y, z\|=1
$$

is satisfied. Clearly, for $t=0$ and $t=1$, the inequality (12) is satisfied. If $t \in(0,1)$, then 1) implies

$$
\begin{aligned}
\left\|\frac{x+y}{2}, z\right\| & =\frac{1-t}{2}\left(1+\frac{t}{1-t}\right)\|x+y, z\| \\
& =\frac{1-t}{2}\left(\|x, z\|+\left\|\frac{t}{1-t} y, z\right\|\right)\left\|\frac{x}{\|x, z\|}-\frac{\frac{t}{t-1} y}{\left\|\frac{t}{t-1} y, z\right\|}, z\right\| \\
& \leq \frac{1-t}{2}\left(\|x, z\|+\left\|\frac{t}{1-t} y, z\right\|\right)\left(2 C \frac{\left\|x-\frac{t}{t-1} y, z\right\|}{\|x, z\|+\left\|\frac{t}{t-1} y, z\right\|}+(C-1) \frac{\max \left\{\|x, z\|,\left\|\frac{t}{t-1} y, z\right\|\right\}}{\|x, z\|+\left\|\frac{t}{t-1} y, z\right\|}\right) \\
& =C(1-t)\left\|x-\frac{t}{t-1} y, z\right\|+\frac{(C-1)(1-t)}{2} \max \left\{1, \frac{t}{1-t}\right\} \\
& =C\|(1-t) x+t y, z\|+\frac{C-1}{2} \max \{1-t, t\},
\end{aligned}
$$

i.e. the inequality (12) holds true.

$2) \Rightarrow 1)$. Let assume that 2) is satisfied. Further, let $x$ and $y$ be arbitrary non-null vectors at $L$. Then for $\frac{x}{\|x, z\|}, \frac{-y}{\|y, z\|} \in L$ it is true that

$$
\left\|\frac{x}{\|x, z\|}, z\right\|=\left\|\frac{-y}{\|-y, z\|}, z\right\|=1
$$

and if $t=\frac{\|y, z\|}{\|x, z\|+\|y, z\|}$, then 2) implies that

$$
\begin{aligned}
\left\|\frac{x}{\|x, z\|}-\frac{y}{\|y, z\|}, z\right\|= & 2\left\|\frac{\frac{x}{\|x, z\|}+\frac{-y}{\|y, z\|}}{2}, z\right\| \\
\leq & 2\left(C\left\|\left(1-\frac{\|y, z\|}{\|x, z\|+\|y, z\|}\right) \frac{x}{\|x, z\|}+\frac{\|y, z\|}{\|x, z\|+\|y, z\|} \cdot \frac{-y}{\|y, z\|}, z\right\|\right. \\
& +\frac{C-1}{2} \max \left\{1-\frac{\|y, z\|}{\|x, z\|+\|y, z\|}, \frac{\|y, z\|}{\|x, z\|+\|y, z\|}\right\} \\
= & 2 C \frac{\|x-y, z\|}{\|x, z\|+\|y, z\|}+(C-1) \frac{\max \{\|x, z\|,\|y, z\|\}}{\|x, z\|+\|y, z\|}
\end{aligned}
$$

i.e. the inequality (11) holds true.

Remark 2. The inequality (11) is actually generalization of the inequality

$$
\left\|\frac{x}{\|x, z\|}-\frac{y}{\|y, z\|}, z\right\| \leq \frac{2\|x-y, z\|}{\|x, z\|+\|y, z\|},
$$


which on 2-normed space, is satisfied if and only if the 2-norm is generated by 2-inner product ([10]). So, it is logically to be stated the following question:

Does the inequality (11) in quasi 2-normed space with modulus of concavity $C \geq 1$ hold true if and only if it exists a function $f: L \times L \rightarrow \mathbf{R}$ so that $f(x, x, z)=\|x, z\|^{2}$.

\section{References}

[1] A. Malčeski and R. Malčeski, Inequalities of Pečarić-Rajić type in quasi 2normed space, IJSIMR, Vol. 3, Issue 5 (2015), 1-7.

[2] A. Malčeski, R. Malčeski, K. Anevska and S. Malčeski, A remark about quasi 2normed space, Applied Mathematical Sciences, Vol. 9, No. 55 (2015), 2717-2727.

[3] C. Park, Generalized quasi-Banach spaces and quasi-(2;p) normed spaces, Journal of the Chungcheong Matematical Society, Vol. 19, No. 2 (2006), 197 206

[4] M. Kir and M. Acikgoz, A study involving the completion of quasi 2-normed space, International Journal of Analysis (2013), http://dx.doi/10.1155.2013/ 512372

[5] R. Malčeski and A. Malčeski, Inequalities of Dunkl-Williams and Mercer type in quasi-normed space, IJSIMR, Vol. 3, Issue 1 (2015), 67 - 70

[6] R. Malčeski and K. Anevska, Families of norms generated by 2-norm, AJER, Vol. 03, Iss. 05 (2014), 315 - 320

[7] R. Malčeski and K. Anevska, Parallelepiped inequality into 2-normed space and its consequences, IJSIMR, Vol. 2, Issue 08 (2014), 719 - 728

[8] R. Malčeski, Inequalities of Pečarić-Rajić type in quasi-normed space, Applied Mathematical Sciences, Vol. 9, no. 15 (2015), 729-737

[9] R. Malčeski, On the Sharp Triangle Inequalities in Quasi-Normed Spaces, British Journal of Mathematics \& Computer Science, Vol. 7, No. 4 (2015), 258-265

[10] S. Malčeski, A. Malčeski, K. Anevska, R. Malčeski, Another characterization's of 2pre-Hilbert Space, IJSIMR, Vol. 3, Issue 2 (2015), 45-54

[11] S. Gähler, Lineare 2-normierte Räume, Math. Nachr. 28 (1965), 1-42

\footnotetext{
${ }^{1)}$ Faculty of informatics, FON University, Skopje, Macedonia

E-mail address: anevskak@gmail.com

${ }^{2)}$ Centre for research and development of education, Skopje, Macedonia

E-mail address: samoil.malcheski@gmail.com
} 